\title{
Experimental evidence of symmetry-breaking supercritical transition in pipe flow of shear-thinning fluids
}

\author{
Chaofan Wen, ${ }^{1}$ Robert J. Poole, ${ }^{1}$ Ashley P. Willis, ${ }^{2}$ and David J. C. Dennis ${ }^{1, *}$ \\ ${ }^{1}$ School of Engineering, University of Liverpool, Liverpool, L69 3GH, United Kingdom \\ ${ }^{2}$ School of Mathematics and Statistics, University of Sheffield, Sheffield, S3 7RH, United Kingdom
}

(Received 25 May 2016; published 6 March 2017)

\begin{abstract}
Experimental results reveal that the asymmetric flow of shear-thinning fluid through a cylindrical pipe, which was previously associated with the laminar-turbulent transition process, appears to have the characteristics of a nonhysteretic, supercritical instability of the laminar base state. Contrary to what was previously believed, classical transition is found to be responsible for returning symmetry to the flow. An absence of evidence of the instability in simulations (either linear or nonlinear) suggests that an element of physics is lacking in the commonly used rheological model for inelastic shear-thinning fluids. These unexpected discoveries raise new questions regarding the stability of these practically important fluids and how they can be successfully modeled.
\end{abstract}

DOI: 10.1103/PhysRevFluids.2.031901

Pipe flow of non-Newtonian fluids is of great practical importance because many manmade fluids are non-Newtonian, for example, cosmetics and food products like shampoos or sauces, and the manufacturing processes for these fluids invariably involve pipe flow. Such fluids often have complicated material properties: Their viscosity can change with the flow rate (shear thinning or shear thickening), stresses created in complex fluids can become strongly anisotropic in space and depend on the deformation history (viscoelasticity), and their simple flows can spontaneously split into several regions moving at different deformation rates (shear banding). In this paper we demonstrate that pipe flows of shear-thinning fluids exhibit an unexpected spontaneous symmetry breaking, leading to asymmetric velocity profiles. We present experimental evidence that this asymmetry is not due to the laminar-turbulent transition process (as previously believed) but has the signature of a supercritical instability.

Scientific interest in the transition from laminar to turbulent flow in a cylindrical pipe can be traced back to the renowned work of Reynolds. One of the primary reasons that the dynamical process of transition in pipe flow is so intriguing is that all theoretical and numerical evidence suggests that laminar pipe flow is linearly stable for all Reynolds numbers $(R e)[1,2]$, yet abrupt transition to turbulence cannot ultimately be avoided. Much progress has been made in recent years through the discovery of exact solutions to the Navier-Stokes equations (in the form of traveling waves) at transitional $R e$ [3-8] and how the footprints of these solutions persist into fully turbulent flows [9].

Although not as widely studied as the pipe flow of Newtonian fluids, pipe flow of non-Newtonian, shear-thinning fluids is also scientifically interesting for the similarities and, significantly, differences between shear thinning and Newtonian flow in transition. Previous work suggests that, like its Newtonian counterpart, pipe flow of simple, inelastic power-law-type shear-thinning fluids is linearly stable [10]. However, the most confusing aspect of the flow of non-Newtonian fluids is the asymmetric form the velocity profile takes at transitional Reynolds numbers $\left(\approx 10^{3}\right.$ to $\left.10^{4}\right)$ in a pipe of circular

\footnotetext{
*djcd@liverpool.ac.uk
}

Published by the American Physical Society under the terms of the Creative Commons Attribution 4.0 International license. Further distribution of this work must maintain attribution to the author(s) and the published article's title, journal citation, and DOI. 
WEN, POOLE, WILLIS, AND DENNIS

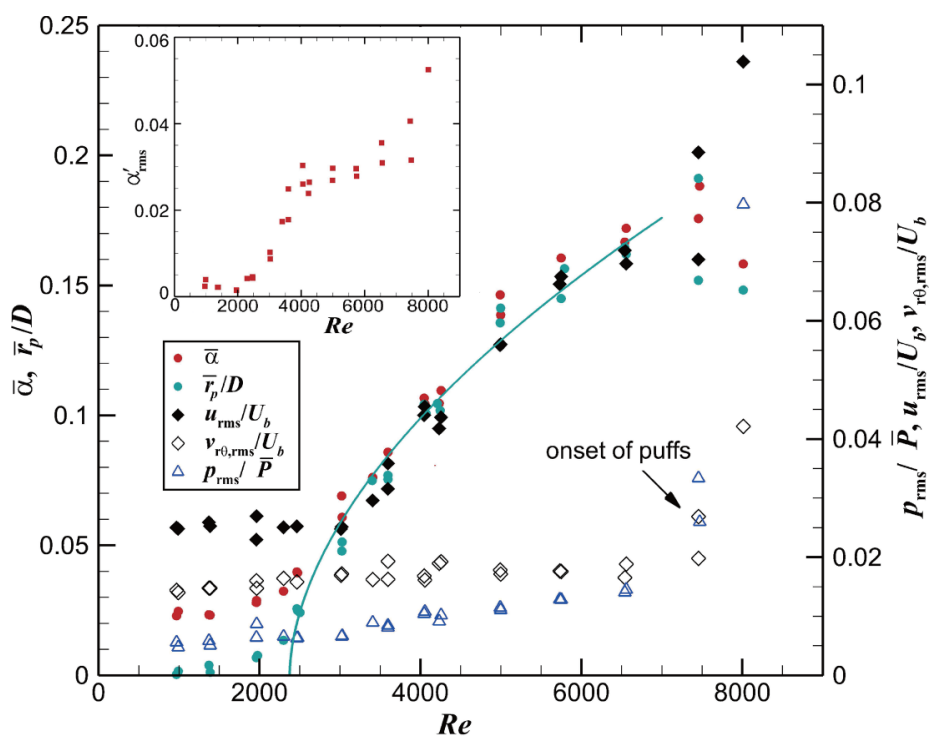

FIG. 1. Variation of asymmetry measures $\bar{\alpha}, \overline{r_{p}} / D$ and $u_{\mathrm{rms}} / U_{b}$ (closed symbols) and transition indicators $p_{\text {rms }} / \bar{P}$ and $v_{r \theta, \text { rms }} / U_{b}$ (open symbols). The solid curve is a power law of the form $\overline{r_{p}} / D=a\left(R e-R e_{c}\right)^{b}$, where $b=0.55$. Inset shows dependence of the fluctuations of $\alpha$ on $R e$.

(and therefore axisymmetric) cross section. This counterintuitive observation remains unexplained despite its ubiquity in all experimental results since its discovery around 20 years ago. Rarely is such an observation made that appears so unphysical, but it has now been found experimentally in independent research programs in the United Kingdom [11,12], France [13-15], Australia [16], and subsequently Canada [17]. The asymmetry has therefore been observed in several facilities using a wide range of shear-thinning fluids, including aqueous solutions of xanthan gum, polyacrylamide, carboxymethylcellulose, carbopol, and laponite [16]. The problem appears universal as it is observed consistently and remains for the whole duration of the experiments (on the order of weeks). Many possible causes have been tested and eliminated, such as the rotation of the earth, curvature of the pipe axis, significant imperfection of flow geometry, upstream and downstream disturbances, temperature gradients, and inlet effects $[12,16]$. The question of what physical mechanism is leading to such an unusual flow remains open and of broad interest given its fundamental nature.

In this work we provide experimental results that simultaneously capture the three-component velocity field across the entire pipe cross section, a significant advancement on the exclusively pointwise single-component measurements of previous works. This leads to a much clearer picture of the behavior of the asymmetric velocity profile and reveals that the asymmetry is produced by a pathway that has not previously been proposed. As shown in Fig. 1, three different bifurcation measures exhibit an approximate square-root dependence, the signature of a supercritical bifurcation, at a Reynolds number far below subcritical transition associated with puffs, indicated by the sudden large increase in the pressure fluctuations, $p_{\text {rms }}$, and cross-stream fluctuations, $v_{r \theta \text {,rms }}$, in Fig. 1 . Thus, it is apparent that the asymmetry observed in pipe flow of shear-thinning fluids is not associated with the laminar-turbulent transition process, but is in fact an instability of the laminar flow. This insight is in direct contradiction with the previous thinking on this matter [11-17].

The experiments were conducted in the Very Large Scale Pipe Flow (VLSPF) facility at the University of Liverpool. The facility comprises a 23.3-m-long pipe constructed of a set of borosilicate glass tubes with $100 \pm 0.1-\mathrm{mm}$ internal diameter. To avoid geometrical imperfections and the possibility of longitudinal curvature effects leading to secondary flow, the pipe was assembled using a laser and target alignment process, which ensures the overall end-to-end straightness well 


\section{EXPERIMENTAL EVIDENCE OF SYMMETRY-BREAKING ...}

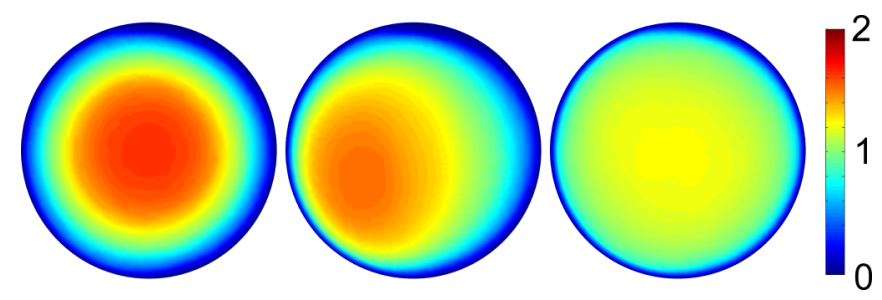

FIG. 2. Time-averaged streamwise velocity profile $\left(U / U_{b}\right)$ for laminar $(R e=300, \bar{\alpha}=0.025)$, transitional $(R e=8000, \bar{\alpha}=0.165)$, and turbulent $(R e=15000, \bar{\alpha}=0.018)$ flow, from left to right, respectively.

within $1 \mathrm{~mm}$. A plenum chamber is positioned before the inlet which includes a complete flow reversal followed by baffles and screens to eliminate any effect the final bend may have on the flow. The flow is investigated using a high-speed, stereoscopic particle image velocimetry (SPIV) technique (similar to Ref. [9]) in which the measurement plane (located $22 \mathrm{~m}$ from the pipe inlet, corresponding to $220 D$ where $D=$ pipe diameter) is perpendicular to the streamwise velocity, providing all three components of velocity across the entire pipe cross section.

The working fluid was a $0.15 \%$ (by weight) aqueous solution of a xanthan gum (Keltrol TF), which exhibits shear-thinning behavior to the extent that (in the shear rate range of interest) an increase by two orders of magnitude in shear rate leads to a decrease by a single order of magnitude in viscosity with no shear banding. In consideration of this inhomogeneity of the viscosity, the Reynolds number is defined as $R e=\rho U_{b} D / \mu_{w}$, where $\rho$ is the density of the working fluid, $U_{b}$ is the bulk velocity, $D$ is the inner diameter of the pipe, and $\mu_{w}$ is the viscosity corresponding to the average shear rate at the wall (calculated using pressure-drop measurements) at the appropriate flow rate. The relationship between shear rate and viscosity is obtained using a controlled-stress rotational rheometer with a cone-and-plate geometry (cone angle $2^{\circ}$ ). In the shear rate range $32<\dot{\gamma}<373 \mathrm{~s}^{-1}$ (which corresponds approximately to the average wall shear rate range in these experiments), this relationship is well fit by a power law of the form $\mu_{w}=k \dot{\gamma}^{n-1}$, with $k=0.165 \mathrm{~Pa} \mathrm{~s}^{n}$ and $n=0.5$.

Figure 2 shows time-averaged streamwise velocity profiles obtained using the SPIV at laminar, transitional, and turbulent Reynolds numbers. Axisymmetric velocity profiles are clearly observed for both laminar and turbulent flow as expected. Indeed, the laminar profile agrees well with that of the analytical solution for fully developed pipe flow of a power-law fluid with the appropriate power-law index. A significant asymmetry is observed at the transitional Reynolds number, which is consistent with observations from previous laser Doppler velocimetry (LDV) measurements [16]. No secondary flow is measurable in the asymmetric state.

An asymmetry parameter, $\alpha$,

$$
\alpha=\frac{\int_{0}^{R} \int_{0}^{2 \pi}\left|U(r, \theta)-U_{M}(r)\right| d \theta r d r}{\int_{0}^{R} U_{M}(r) 2 \pi r d r},
$$

is introduced to quantify the degree of azimuthal flow asymmetry in any given snapshot and reveal how the asymmetry varies in time. In Eq. (1), $U_{M}$ is the arithmetic mean of the streamwise velocity $U$ at the specified radial location $r$ along the circumferential direction as defined in Eq. (2),

$$
U_{M}(r)=\frac{\int_{0}^{2 \pi} U(r, \theta) d \theta}{2 \pi} .
$$

The values of $\bar{\alpha}$ given for the laminar (2.5\%) and turbulent (1.8\%) flows are associated with the random error in the SPIV measurements, whereas in the asymmetric case it is an order of magnitude higher $(16.5 \%)$.

One of the major insights provided by our SPIV measurements in comparison to all other previous work is the time-varying behavior of the asymmetric flow pattern, which was hitherto considered stationary. The variation in time is highlighted in Fig. 3 by the time history of the 


\section{WEN, POOLE, WILLIS, AND DENNIS}

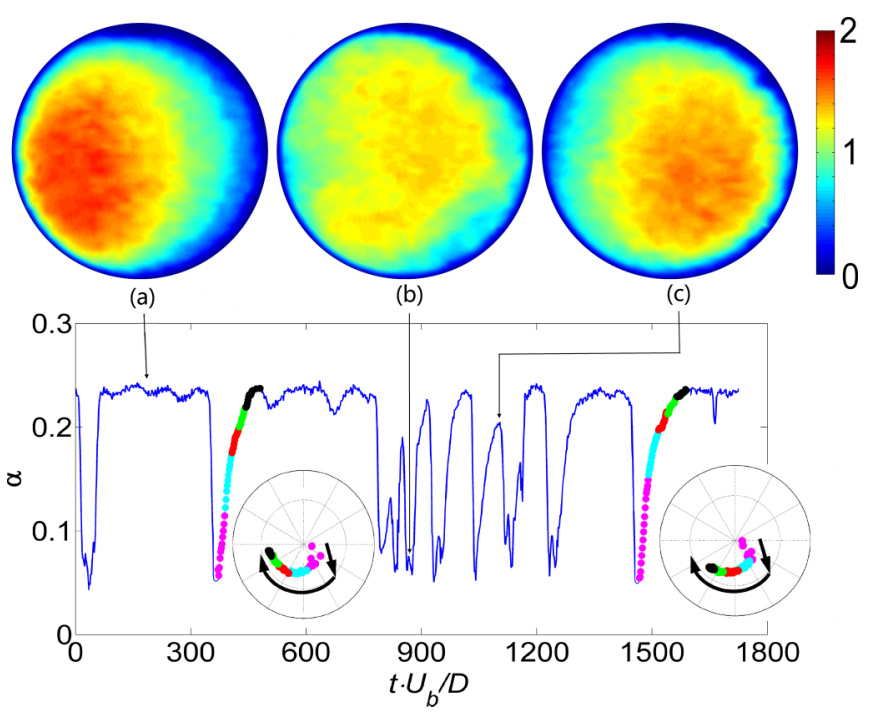

FIG. 3. The time-varying nature of the asymmetric flow pattern at $R e=8000$ is shown by the time history of asymmetry factor, $\alpha$ (bottom) and instantaneous cross-stream snapshots of the streamwise velocity $\left(U / U_{b}\right)$. (a) Asymmetric flow with preferred orientation, (b) temporarily quasiaxisymmetric flow induced by a turbulent puff, and (c) a brief visit to asymmetric flow with an alternative orientation. The experimental duration is approximately $200 \mathrm{~s}$ and the SPIV acquisition rate is $5 \mathrm{~Hz}$. The two insets show the location of the peak velocity in the radial-azimuthal plane as the asymmetry returns following the passing of a puff.

asymmetry factor $(\alpha)$ showing frequent dramatic drops, indicating that the flow is briefly returning to a quasiaxisymmetric state [an example is shown in Fig. 3(b)]. These events are associated with turbulent puffs passing through the measurement plane. A turbulent puff is captured using high-speed SPIV and pseudospatially reconstructed using Taylor's approximation $([18,19]$ using the bulk velocity, $U_{b}$ as the convection velocity) in Fig. 4. It is clear from the simultaneous time histories of nondimensional swirling strength $\left(\overline{\lambda_{c i}} D / U_{b}\right.$, where $\overline{\lambda_{c i}}$ is the imaginary part of the complex eigenvalue of the 3D velocity gradient tensor averaged across the pipe cross section) and $\alpha$ that the presence of a turbulent puff (indicated by high swirling strength) leads to a sudden decrease in the asymmetry factor, signaling that the flow pattern is becoming much more axisymmetric.

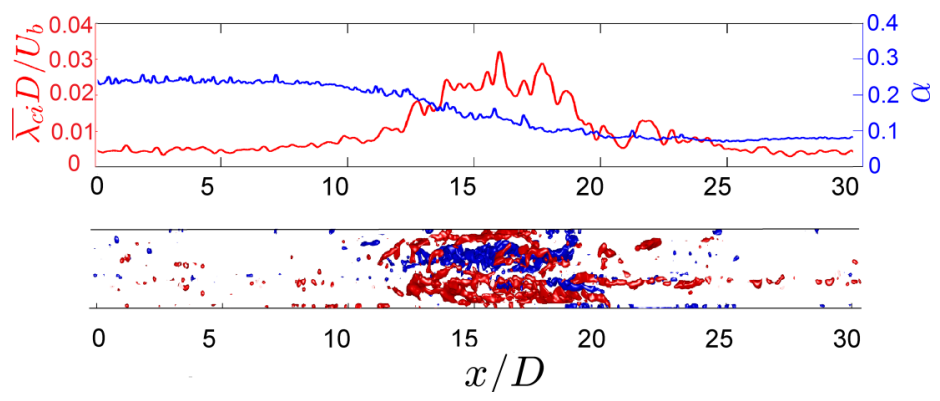

FIG. 4. The temporary elimination of the asymmetry by a turbulent puff at $R e=8000$. The top panel shows simultaneous time histories of the nondimensional swirling strength and the asymmetry factor $(\alpha)$. The bottom panel shows a visualization of a puff using isosurfaces of swirling strength $\left(\lambda_{c i} D / U_{b}=0.012\right)$; red and blue indicate regions on the isosurface where the local axial vorticity is positive and negative respectively. The SPIV acquisition rate is $200 \mathrm{~Hz}$. 
Returning to Fig. 3, the example asymmetric flow pattern shown in Fig. 3(a) is the preferred flow pattern in which the flow spends most of its time and therefore has a profound influence on the asymmetric time-averaged velocity profile seen in Fig. 2. We have confirmed that this favored location is largely invariant with axial location by taking LDV measurements at many locations (over several meters) upstream of the SPIV measurement plane and finding little change in the orientation of the asymmetry. The invariance of the orientation with axial location indicates that the asymmetry is not due to a helical instability spiralling down the pipe. It is also notable that previous work examined the effect of inlet disturbance on the asymmetry by obstructing the flow in the plenum chamber just before the inlet on the side of the pipe where the highest velocity of asymmetry appeared [12]. The blockage introduced an asymmetric disturbance with a different orientation into the flow. However, this imposed asymmetry was not sustained through the development of flow, and the asymmetry at the measurement plane $(220 \mathrm{D}$ from inlet) recovered to the same favored location as that in the unblocked case, demonstrating the robustness of the asymmetry and its favored location.

It is clear from the instantaneous and time-averaged values of $\alpha$ that the flow is instantaneously more asymmetric than any other previous measurements have indicated $(\alpha \approx 23 \%)$. Although the asymmetric flow pattern is most commonly observed in this orientation, there are numerous brief instances when the orientation is different. One example is indicated in Fig. 3(c). This is from an instance when the flow is returning to asymmetry after the passing of a turbulent puff. It is commonly seen that immediately following a puff the asymmetry reappears in a different orientation and then slowly rotates to its preferred location as shown in the insets in Fig. 3.

Given that the velocity profile in both the laminar and turbulent flow regimes is axisymmetric and in between it is found to be asymmetric, an important question is precisely how the velocity profile varies with Reynolds number. In particular, how does the asymmetry first form and how does the flow axisymmetry return at higher $R e$ ? We answer these questions by taking SPIV measurements across a range of Reynolds numbers from laminar through to transitional Re. Figure 1 shows that beyond a critical Reynolds number $\left(R e_{c}\right)$ the magnitude of the asymmetry factor $(\bar{\alpha})$ increases steadily with $R e$. In fact, it approximately grows with the square root of $R e$, suggesting a supercritical bifurcation. The radius at which the peak in the profile of the streamwise velocity is located is another measure of the flow asymmetry (i.e., an alternative bifurcation parameter). Here we define $r_{p}$ as the flow-weighted mean radius of the fastest $20 \%$ of the flow (a more robust measure than simply the instantaneous maximum of $U$ ) and plot its time average in Fig. 1 normalized by the pipe diameter $\left(\overline{r_{p}} / D\right)$. It also has an approximately square-root dependence on $R e$ : Fitting a power law of the form $\overline{r_{p}} / D=a\left(R e-R e_{c}\right)^{b}$ yields $b=0.55$ (with an $R$-squared value of 0.9854 ), which has been plotted as a curve on Fig. 1 .

It is well known that classical transition to turbulence in many parallel shear flows, including Newtonian pipe flow, is a subcritical rather than a supercritical phenomenon and therefore the square-root behavior of the asymmetry measures $\left(\alpha\right.$ and $\left.r_{p} / D\right)$ indicates that the asymmetry is not $d u e$ to the laminar-turbulent transition process. To determine the $R e$ at which classical transition to turbulence (in the form of puffs) is occurring, we can examine the pressure and velocity fluctuations. In Fig. 1 we plot root mean square (rms) of the pressure fluctuations normalized by mean pressure $\left(p_{\text {rms }} / \bar{P}\right)$. The square-root behavior is not observed and instead we see a sudden increase in the pressure fluctuations (i.e., subcritical transition) at $R e=7000-8000$ (within the expected range for shear-thinning fluids, which are known to delay transition to turbulence in comparison to Newtonian flows [20]), significantly higher than the $R e$ at which the supercritical bifurcation is observed $(\operatorname{Re} \approx 2300)$.

The streamwise velocity fluctuations (plotted normalized by bulk velocity in Fig. 1 as $u_{\mathrm{rms}} / U_{b}$ ) follow the trend of the asymmetry measures with a square-root dependence on $R e$, whereas the velocity fluctuations in the radial-azimuthal $(r-\theta)$ plane follow a trend similar to that of the pressure fluctuations. Turbulence in pipe flow is inherently three-dimensional and so all components of the velocity should show substantial fluctuations when transition occurs. The reason that the streamwise component follows the trend of the asymmetry measures is due to the fact that beyond the onset of the instability it is apparent that the asymmetry is not stationary in time; it therefore creates a 


\section{WEN, POOLE, WILLIS, AND DENNIS}

fluctuation in $U$ due to this movement, which is not seen in the in-plane velocity components because the asymmetry is not seen in those components and there is no significant secondary flow associated with the asymmetry.

Varying the concentration of xanthan gum in the mixture can be used to vary the magnitude of the viscosity dependence on shear (which can be broadly quantified using the power-law parameter, $n$ ). A direct relationship between shear thinning and the magnitude of the asymmetry is observable, reinforcing the idea that the instability is strongly dependent on the shear-thinning nature of the fluid and is commensurate with the fact that the asymmetry is never observed in Newtonian fluid flow. Continuation of the power law parameter towards $n=1$ is heading towards the Newtonian case, and indeed the magnitude of the asymmetry gradually decreases and disappears at $n=1$. Furthermore, all mixtures exhibit very similar behavior, particularly that the asymmetry measures grow with an approximately square-root dependence on $R e$.

Simulations have been performed using an extension of the openpipeflow.org Navier-Stokes solver for the experimental parameters, with an inelastic Carreau-Yasuda rheology matching the shear-thinning characteristics of the working fluid in the experiments. These simulations in a periodic domain of length $15 D$ reproduce subcritical turbulence for $R e \gtrsim 6500$, as observed in the experiments. Below these values of $R e$, however, perturbations return to the axisymmetric state. The Coriolis effect has been examined as an origin for the asymmetry, but in simulations was found to induce only small $\alpha \approx 0.01$ at $R e=4500$. This might, however, be sufficient to excite another (the experimentally observed) instability with preferential orientation. Thus far the numerical simulations have not shown the symmetry-breaking bifurcation seen in the experiments. This indicates that the current simulations have not incorporated all of the relevant physics associated with the complex working fluid. For example, mild elasticity may be important. However, it is nontrivial to correctly identify the relevant constitutive equation that should be used in this case. This also explains why previous theoretical work considering a purely shear-thinning (power law) inelastic fluid found it to be linearly stable [10], whereas our experiments imply it is unstable. It appears that modeling the shear-thinning characteristics alone is insufficient to capture the instability.

The inset of Fig. 1 shows the fluctuations in the asymmetry factor $\left(\alpha_{\mathrm{rms}}^{\prime}\right)$. They show that close to the bifurcation $\left(R e \gtrsim R e_{c}\right) \alpha$ is very nearly steady (as the magnitude of $\alpha_{\text {rms }}^{\prime}$ is comparable to the measurement noise) but as $R e$ increases the fluctuations in $\alpha$ increase significantly. These fluctuations can also be observed in the time history of $\alpha$ shown in Fig. 3. This indicates that with increasing $R e$, not only does the magnitude of asymmetry evolve, but the flow also manifests a more pronounced time-varying nature. If the instability is a Hopf bifurcation we would expect a small-amplitude limit cycle to occur just above the Reynolds number at which the instability appears. We cannot identify a single frequency at the onset of the instability in our experimental data, probably because the amplitude of the oscillation is too small to be measured with the SPIV. However, a little further above $\operatorname{Re}_{c}(2500<R e<3500)$ we observed oscillations across a range of low frequencies $(f<0.1 \mathrm{~Hz})$ in $\alpha, r_{p}$, and $U$. These frequencies correspond reasonably well with those found by Esmael et al. [15], once they are converted into Strouhal number $\left(S t=f D / U_{b} \approx 10^{-3}\right)$. We also observed a power law decay in the energy spectra of the axial velocity fluctuations with an exponent of approximately -3 , which again is similar to that found by Esmael et al. [15]. Due to the limitations of their experiment (single-point measurements of one component of velocity), Esmael et al. [15] were not able to identify a supercritical bifurcation and interpreted their results as indicating a weakly turbulent state, which was chaotic in time and regular in space, induced by the shear-thinning behavior of the fluid. While our results are entirely consistent with their data, our interpretation is quite different as there is now more evidence to suggest the presence of a supercritical instability.

Figure 5 shows the path that the asymmetry takes to reach its favored orientation as the Reynolds number is varied. Each dot corresponds to the location of the peak velocity at an instant in time. Each color corresponds to a nominal step change in $R e$, although it should be noted that throughout the whole duration of the experiment the flow is transiently changing as at no point does it reach a true steady state. Figure 5 shows that, remarkably, the asymmetry is nonhysteretic and reversible: It has 


\section{EXPERIMENTAL EVIDENCE OF SYMMETRY-BREAKING ...}
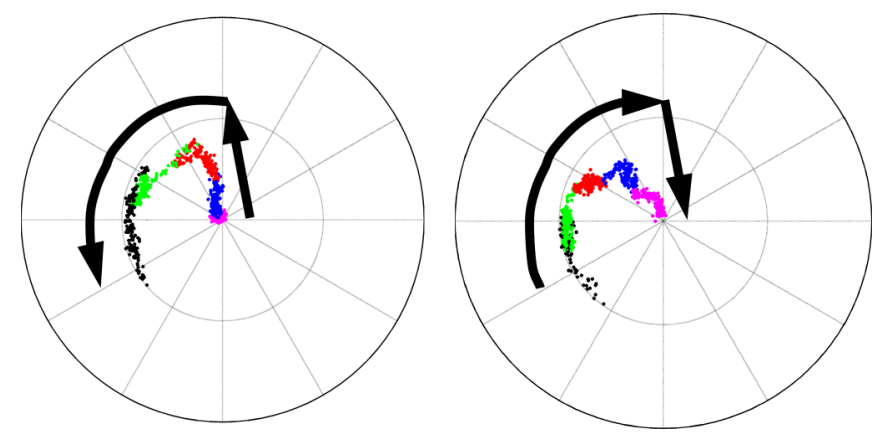

FIG. 5. Traces of the peak velocity during the transient processes of axisymmetric-asymmetric transition (left) and asymmetric-axisymmetric relaminarisation (right). Each dot corresponds to an instant in time color coded by its nominal $R e$ according to $R e=1960$ (pink), $R e=3400$ (blue), $R e=4230$ (red), $R e=5750$ (green), and $R e=6740$ (black) with the time delay between each $R e$ being $30 \mathrm{~s}$. The arrows indicate the direction of movement with time.

a preferred route between axisymmetry and asymmetry, which it adheres to regardless of whether it is approaching transition to turbulence or relaminarizing. The similarity between Fig. 5 and the insets of Fig. 3 is clear. Broadly speaking, the asymmetry moves outward from the center of the pipe and then rotates to its preferred azimuthal location along an approximately constant radius.

The experimental results presented in this paper demonstrate that a counterintuitive, yet apparently universal, asymmetry in pipe flow of shear-thinning fluids is not caused by the laminar-turbulent transition process (as previously believed) but is a distinct supercritical instability of the laminar base flow, which is in fact eliminated by the turbulent structures that are formed during classical subcritical, laminar-turbulent transition. We have therefore identified a flow instability that is unique to shear-thinning fluids.

The square-root dependence of the symmetry breaking with Re suggests a pitchfork. For this geometry a pitchfork of revolution might be expected, meaning any orientation is possible in principle. However, any imperfection in the experiment (including the relatively small effect of the Coriolis force) may break the symmetry of the flow, making one orientation (i.e., solution) stable and all others unstable [21,22], thus dictating the particular favored orientation observed in the experiments. The robustness of the preferred orientation of the asymmetry in the experiments is consistent with this idea. A supercritical Hopf bifurcation is also possible, given that its frequency signal quite likely would be within the experimental noise at onset. The simulations suggest that a subcritical instability is unlikely as any nonlinear perturbation, including in the presence of a small Coriolis force, is found to decay. Together with the experiments, these results imply that laminar pipe flow of real shear-thinning fluids may be linearly unstable to asymmetric disturbances, which is not something that has previously been considered and presents many questions for all researchers interested in the stability of fluid flows.

The authors wish to acknowledge fruitful discussions with R. R. Kerswell, M. D. Graham, X. Wu, and A. Morozov. C.W. was funded by the China Scholarship Council. R.J.P. acknowledges funding for a fellowship in complex fluids and rheology from the Engineering and Physical Sciences Research Council (EPSRC, UK) under Grant No. EP/M025187/1.

[1] B. Eckhardt, T. M. Schneider, B. Hof, and J. Westerweel, Turbulence transition in pipe flow, Ann. Rev. Fluid Mech. 39, 447 (2007).

[2] T. Mullin, Experimental studies of transition to turbulence in a pipe, Ann. Rev. Fluid Mech. 43, 1 (2011). 


\section{WEN, POOLE, WILLIS, AND DENNIS}

[3] H. Faisst and B. Eckhardt, Traveling Waves in Pipe Flow, Phys. Rev. Lett. 91, 224502 (2003).

[4] H. Wedin and R. R. Kerswell, Exact coherent structures in pipe flow: Travelling wave solutions, J. Fluid Mech. 508, 333 (2004).

[5] B. Hof, C. W. H. van Doorne, J. Westerweel, F. T. M. Nieuwstadt, H. Faisst, B. Eckhardt, H. Wedin, R. R. Kerswell, and F. Waleffe, Experimental observation of nonlinear traveling waves in turbulent pipe flow, Science 305, 1594 (2004).

[6] A. P. Willis and R. R. Kerswell, Coherent Structures in Localized and Global Pipe Turbulence, Phys. Rev. Lett. 100, 124501 (2008).

[7] A. de Lozar, F. Mellibovsky, M. Avila, and B. Hof, Edge State in Pipe Flow Experiments, Phys. Rev. Lett. 108, 214502 (2012).

[8] M. Chantry, A. P. Willis, and R. R. Kerswell, Genesis of Streamwise-Localized Solutions from Globally Periodic Traveling Waves in Pipe Flow, Phys. Rev. Lett. 112, 164501 (2014).

[9] D. J. C. Dennis and F. M. Sogaro, Distinct Organizational States of Fully Developed Turbulent Pipe Flow, Phys. Rev. Lett. 113, 234501 (2014).

[10] N. S. López-Carranza, M. Jenny, and C. Nouar, Pipe flow of shear-thinning fluids, C. R. Mec. 340, 602 (2012).

[11] M. P. Escudier and F. Presti, Pipe flow of a thixotropic liquid, J. Non-Newtonian Fluid Mech. 62, 291 (1996).

[12] M. P. Escudier, S. Rosa, and R. J. Poole, Asymmetry in transitional pipe flow of drag-reducing polymer solutions, J. Non-Newtonian Fluid Mech. 161, 19 (2009).

[13] J. Peixinho, C. Nouar, C. Desaubry, and B. Théron, Laminar transitional and turbulent flow of yield stress fluid in a pipe, J. Non-Newtonian Fluid Mech. 128, 172 (2005).

[14] A. Esmael and C. Nouar, Transitional flow of a yield-stress fluid in a pipe: Evidence of a robust coherent structure, Phys. Rev. E 77, 057302 (2008).

[15] A. Esmael, C. Nouar, A. Lefèvre, and N. Kabouya, Transitional flow of a non-Newtonian fluid in a pipe: Experimental evidence of weak turbulence induced by shear-thinning behavior, Phys. Fluids 22, 101701 (2010).

[16] M. P. Escudier, R. J. Poole, F. Presti, C. Dales, C. Nouar, C. Desaubry, L. Graham, and L. Pullum, Observations of asymmetrical flow behavior in transitional pipe flow of yield stress and other shear-thinning liquids, J. Non-Newtonian Fluid Mech. 127, 143 (2005).

[17] B. Güzel, T. Burghelea, I. A. Frigaard, and D. M. Martinez, Observation of laminar-turbulent transition of a yield stress fluid in Hagen-Poiseuille flow, J. Fluid Mech. 627, 97 (2009).

[18] G. I. Taylor, The spectrum of turbulence, Proc. R. Soc. London, Ser. A 164, 476 (1938).

[19] D. J. C. Dennis and T. B. Nickels, On the limitations of Taylor's hypothesis in constructing long structures in a turbulent boundary layer, J. Fluid Mech. 614, 197 (2008).

[20] M. P. Escudier, F. Presti, and S. Smith, Drag reduction in the turbulent pipe flow of polymers, J. NonNewtonian Fluid Mech. 81, 197 (1999).

[21] M. Golubitsky and D. G. Schaeffer, Singularities and Groups in Bifurcation Theory, Vol. 1, Applied Mathematical Sciences Vol. 51 (Springer-Verlag, New York, 1985).

[22] T. Mullin, J. R. T. Seddon, M. D. Mantle, and A. J. Sederman, Bifurcation phenomena in the flow through a sudden expansion in a circular pipe, Phys. Fluids 21, 014110 (2009). 